\title{
Meta-Analysis of the Efficacy and Safety of Imidafenacin for Overactive Bladder Induced by Benign Prostatic Hyperplasia in Men Receiving Alpha-Blocker Therapy
}

\author{
Tong Cai ${ }^{1,2, *}$, Ning Wang ${ }^{2, *}$, Liye Liang ${ }^{3}$, Zhongbao Zhou ${ }^{2}$, Yong Zhang ${ }^{4}$, Yuanshan Cui ${ }^{2,4}$ \\ ${ }^{1}$ School of Clinical Medicine, Binzhou Medical University, Yantai, Shandong, China \\ ${ }^{2}$ Department of Urology, The Affiliated Yantai Yuhuangding Hospital of Qingdao University, Yantai, Shandong, China \\ ${ }^{3}$ Department of Urology, Rizhao traditional Chinese Hospital, Rizhao, Shandong, China \\ ${ }^{4}$ Department of Urology, Beijing Tiantan Hospital, Capital Medical University, Beijing, China
}

\begin{abstract}
Purpose: The aim of this meta-analysis was to evaluate the efficacy and safety of imidafenacin for overactive bladder (OAB) induced by benign prostatic hyperplasia (BPH) in men receiving alpha-blocker monotherapy.

Methods: We performed a systematic research of the PubMed, Embase, and Cochrane Library databases, and searched for studies about alpha-blocker with or without imidafenacin treatment for OAB in patients with $\mathrm{BPH}$. We also investigated the original references of the included texts.

Results: Four randomized controlled trials including 779 participants with BPH (389 in the alpha-blocker+imidafenacin group and 390 in the alpha-blocker only group) were studied. The main efficacy endpoint was the Overactive Bladder Symptom Score, which showed a mean difference of -1.88 (95\% confidence interval, -2.32 to $-1.44 ; \mathrm{P}<0.00001$ ), suggesting that alpha-blocker and imidafenacin treatment was effective in treating men with $\mathrm{OAB}$. As other primary efficacy end points, the International Prostate Symptom Score (IPSS) total score $(\mathrm{P}=0.47)$, the IPSS storage symptom score $(\mathrm{P}=0.07)$, the IPSS voiding symptom score $(\mathrm{P}=0.60)$, and the IPSS quality of life score $(\mathrm{P}=0.18)$ indicated that 2 methods had no significant differences in treating men with $\mathrm{OAB}$. In terms of safety, which was assessed using postvoid residual volume $(\mathrm{P}=0.05)$ and maximum flow rate $(\mathrm{P}=0.53)$, the analysis suggested that combination treatment was very well tolerated.

Conclusions: This study suggested that imidafenacin plus alpha-blocker was an efficacious and safe treatment for OAB symptoms in $\mathrm{BPH}$ patients.
\end{abstract}

Keywords: Imidafenacin; Alpha-blocker; Urinary bladder, Overactive; Prostatic hyperplasia; Lower urinary tract symptoms; Randomized controlled trial

- Fund/Grant Support: This work was supported by the National Nature Science Foundation of China (Nos. 81870525; 81801429), Beijing Municipal Administration of Hospitals' Ascent Plan, Code: DFL20190502 and Beijing Municipal Administration of Hospitals Clinical Medicine Development of Special Funding Support, Code: ZYLX201820.

- Conflict of Interest: No potential conflict of interest relevant to this article was reported.

\section{INTRODUCTION}

As one of the most common conditions that cause lower uri- nary tract symptoms (LUTS), benign prostatic hyperplasia (BPH) often causes urinary tract dysfunction in men aged 50 years and older, with a prevalence of $19 \%-30 \%[1,2]$. The Inter-

Corresponding author: Yuanshan Cui (iD) https://orcid.org/0000-0002-6688-449X Department of Urology, Beijing Tiantan Hospital, Capital Medical University, No.119 South 4th Ring West Road, Fengtai District, Beijing 100070, China E-mail: doctorcuiys@163.com

*Tong Cai and Ning Wang contributed equally to this study as co-first authors. Submitted: May 6, 2020 / Accepted after revision: June 16, 2020 
national Continence Society defined overactive bladder (OAB) as urinary urgency with urinary frequency, nocturia, and sometimes urgency incontinence. In the course of everyday life, $\mathrm{OAB}$ is the most inconvenient symptom for men with $\mathrm{BPH}$ [3]. At present, in order to relieve the symptoms of $\mathrm{BPH}$, the main treatment pattern is drug therapy, especially alpha-blockers. Currently, alpha-blockers are widely used as the first-line therapy for $\mathrm{BPH}$, but it is difficult to improve the storage symptoms of $\mathrm{OAB}$ with alpha-blockers alone. However, anticholinergic agents have been recommended to treat the symptoms of $\mathrm{OAB}$, as they have been found to be efficient in alleviating storage symptoms for men with $\mathrm{BPH}$ [4-7].

Imidafenacin, a selective antimuscarinic drug, was proposed to treat the symptoms of OAB. Recent studies have shown that imidafenacin can decreased urine volume via suppression of Cfibers and reduced nocturnal urine volume for $\mathrm{OAB}$ patients [8]. In some studies, anticholinergics combined with alphablockers have shown demonstrable effects in treating $\mathrm{OAB}$ in men with BPH, but their clinical effect is limited [9]. In clinical practice, there have been concerns that anticholinergic agents are infrequently used to treat $\mathrm{BPH}$ in patients because the inhibitory effect of anticholinergics, which produce detrusor muscle contractions that aggravate urinary symptoms, may increase the risk of the numbers of adverse events or acute urinary retention. Urinary retention can reduce patients' quality of life (QoL). Research has shown an incidence of 68 per 10,000 person-years among men [10]. Dry mouth, as one of the most common and problematic side effects, can often lead to discontinuation of anticholinergic therapy [11]. On the basis of the 2019 European Association of Urology Guideline, patients with $\mathrm{BPH}$ who have $\mathrm{OAB}$ symptoms may use anticholinergic agents simultaneously with an alpha-blocker. Meanwhile, some randomized controlled trials (RCTs) and other studies have sought to demonstrate the effects of anticholinergic agents [12-14].

In order to evaluate the curative effect and safety of combined alpha-blocker and imidafenacin treatment for persistent $\mathrm{OAB}$ associated with $\mathrm{BPH}$, we performed a systematic review and meta-analysis of RCTs.

\section{MATERIALS AND METHODS}

\section{Information Sources and Literature Search}

We systematically retrieved articles from the PubMed, Embase, and the Cochrane Library databases published before January 2020, and searched for studies about alpha-blocker with or without imidafenacin treatment for $\mathrm{OAB}$ in patients with $\mathrm{BPH}$. We searched using the following keywords: "alpha-blocker," "imidafenacin," "RCT," "BPH," "male-LUTS" and "OAB." We also examined original references of the texts that were included.

\section{Inclusion Criteria}

RCTs were included if they met the following criteria: (1) the RCT was topically relevant to this meta-analysis (i.e., it analyzed alpha-blocker with or without imidafenacin treatment for $\mathrm{OAB}$ symptoms resulting from $\mathrm{BPH}$ ); (2) the full text was available and the study was an RCT; and (3) similar observation indicators were used to compare the alpha-blocker+imidafenacin group and the alpha-blocker only group in every RCT.

The following studies were excluded: (1) those that were not RCTs, such as abstracts, reviews, or comments; and (2) those that involved catheterization or patients with a prior diagnosis of neurogenic bladder.

If a duplicate study had been published in different journals or at different time points, we included only the most recently published study. However, every study was included if different measures were evaluated. Fig. 1 presents a flow chart of the selection process for the study.

\section{Quality Assessment}

The Jadad scale was used to determine the quality of the re-

91 Articles were identified including:
PubMED: 68 articles
EMBASE: 11 articles
Cochrane Library databases: 12 article

On the basis of titles and abstracts, 84 articles were excluded.

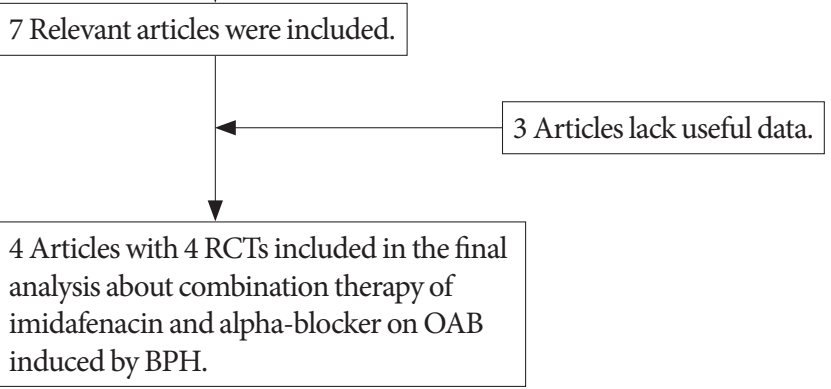

Fig. 1. Flowchart of the study selection process. RCT, randomized controlled trial; $\mathrm{OAB}$, overactive bladder; $\mathrm{BPH}$, benign prostatic hyperplasia. 
trieved RCTs [15]. In the meta-analysis, several metrics were used to assess the quality of the individual studies, including the assignment method of patients, the concealment of allocation, the blinding method, the results of loss to follow-up, and whether there was a calculation of sample size or intention-totreat analysis. The studies were then classified qualitatively using the guidelines published in the Cochrane Handbook for Systematic Reviews of Interventions v.5.1.0 [16]. The quality of every RCT was evaluated as follows: (1) if the study satisfied almost all of the quality criteria, it was considered to have a low risk of bias; (2) if the article partially satisfied the quality criteria or was ambiguous, it would be considered to have a moderate risk of bias; (3) if the quality criteria were rarely met or not described, an article was considered to have a high risk of bias. All authors took part in the quality assessment of each text, and every author agreed with all outcomes. Any differences in opinion regarding the RCTs were resolved through discussion among the authors. All studies were evaluated by all reviewers independently to determine whether they satisfied the inclusion criteria.

\section{Data Extraction}

One author read the articles and collected the following data from studies: (1) the common data in the study(e.g., the baseline demographic and clinical characteristics of study participants); (2) the name of the RCT and authors; (3) the research design and sample size in the study (e.g., the interventions in the different groups; the prostate volume was greater than 16 $\mathrm{mL}$ in the 4 RCTs that were included, and there were no significant differences between the alpha-blocker+imidafenacin group and the alpha-blocker only group in any of the RCTs); (4) data on efficacy in terms of changes in the following parameters, such as total Overactive Bladder Symptom Score (OABSS), the International Prostate Symptom Score (IPSS) total score, the IPSS storage symptoms score, the IPSS voiding symptoms score, the IPSS QoL score, postvoid residual volume (PVR), and maximum flow rate (Qmax). Finally, another author checked the data that we retrieved from the texts. Meanwhile, our team cross-checked the reference documents and data for each study, in order to maintain the integrity of the meta-analysis and the absence of overlapping data in the meta-analysis.

\section{Statistical Analysis and Meta-Analysis}

This meta-analysis of the data was carried out using the RevMan ver. 5.3.0 (Cochrane Collaboration, Oxford, UK) [16]. In order to evaluate the difference between the start and the endpoint in each RCT, our team analyzed changes in the total OABSS, the IPSS total score, the IPSS storage symptoms score, the IPSS voiding symptoms score, the IPSS QoL score, PVR, and Qmax. The mean difference (MD) was used to evaluate continuous data, and odds ratios (ORs) with $95 \%$ confidence intervals (CIs) were calculated to evaluate dichotomous data. A fixed-effects model was used a P-value $>0.05$ was considered to indicate homogeneity. We utilized the I2 statistic to analyze inconsistent results, as this statistic can reflect the proportion of heterogeneity across trials. For this meta-analysis, it was not necessary to obtain ethical approval and patient consent because all the data were acquired from articles that had already been published. A random-effects model was used when the results showed $\mathrm{P}<0.05$ and $\mathrm{I} 2>50 \%$. Moreover, if the results of the study showed a P-value $<0.05$, the result was deemed to be statistically significant.

\section{RESULTS}

\section{Characteristics of the Individual Studies}

We searched for 91 original studies in commonly used databases. On the basis of the aforementioned inclusion and exclusion criteria, 84 studies were excluded. Three other studies were excluded because they lacked informative data. Thus, our final analysis included 4 RCTs [17-20]. The 4 studies included 779 patients (389 in the alpha-blocker+imidafenacin group and 390 in the alpha-blocker only group). A detailed flowchart showing the selection process is shown in Fig. 1. Table 1 displays the baseline characteristics of the studies.

\section{Quality of the Individual Studies}

All 4 RCTs had double-blind processes. All of the RCTs that we found explained their randomization process. Each of the 4 RCTs carried out a calculation to determine the optimal sample size (Table 2). Table 2 demonstrates the quality of each included study. In Fig. 2, a funnel plot displays a qualitative estimation of publication bias for each RCT.

\section{Efficacy}

OABSS

Three RCTs containing 558 participants (279 in the alphablocker+imidafenacin group and 279 in the alpha-blocker only group) measured changes in the OABSS. A fixed-effects model was used to evaluate changes between the alpha-blocker+imidafenacin 


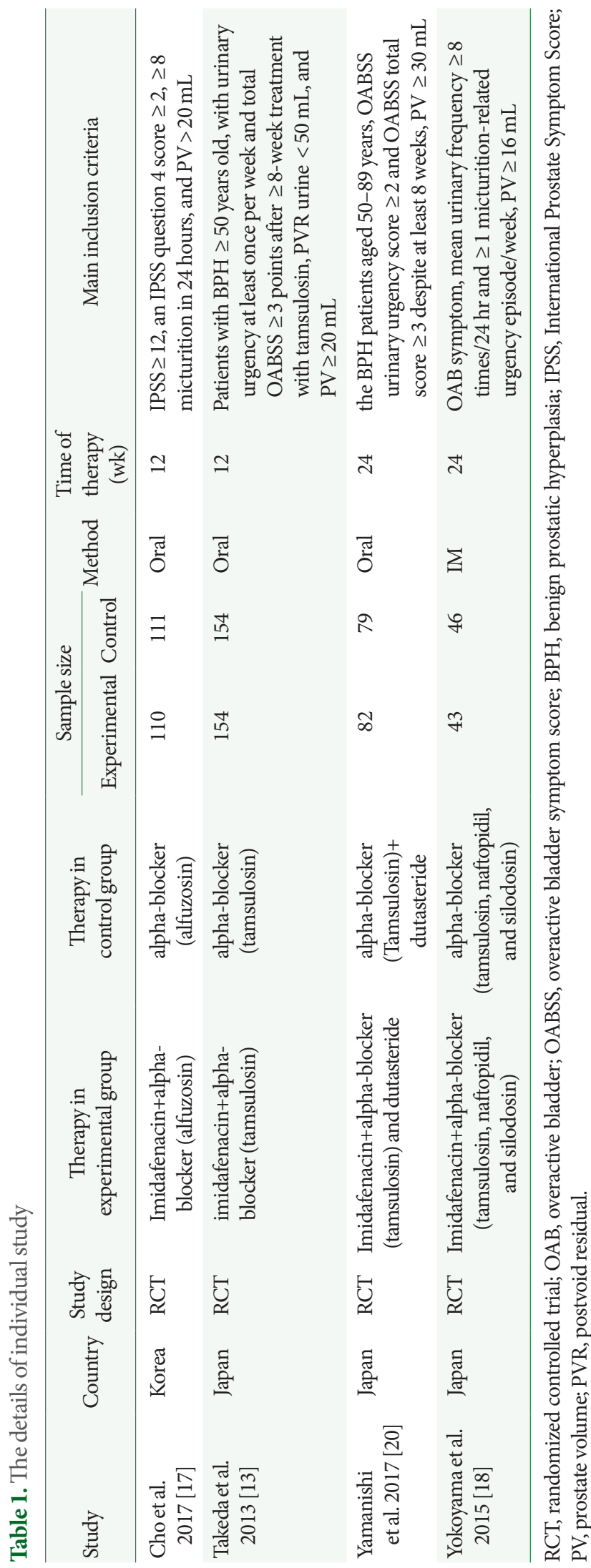

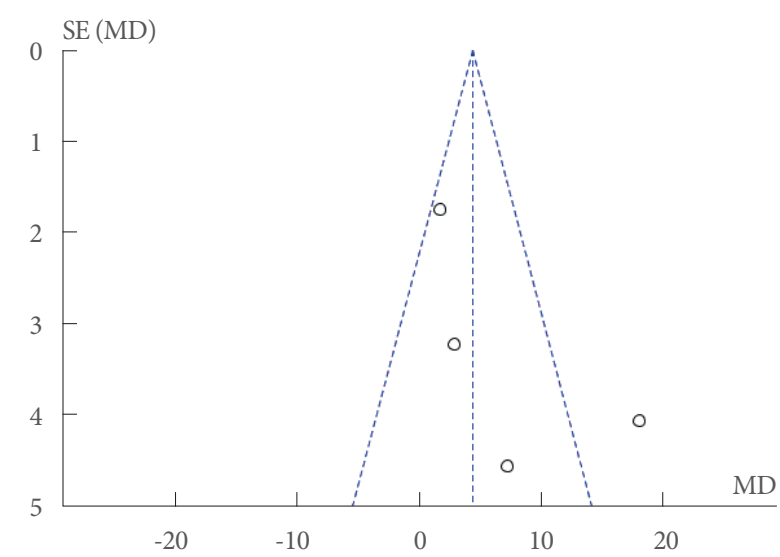

Fig. 2. Funnel plot of the studies included in our meta-analysis. $\mathrm{MD}$, mean difference; SE, standard error.

group and the alpha-blocker only group, showing an $\mathrm{MD}$ of -1.88 (95\% CI, -2.32 to $-1.44 ; \mathrm{P}<0.00001$ ). This finding demonstrates that, compared with the imidafenacin group (Fig. 3), the alphablocker+imidafenacin group showed a statistically significant greater reduction in the OABSS.

\section{IPSS total score}

Four RCTs containing 779 participants (389 in the alphablocker+imidafenacin group and 390 in the alpha-blocker only group) were included. A random-effects model was chosen to evaluate changes between the alpha-blocker+imidafenacin group and the alpha-blocker only group, showing an MD of -0.77 (95\% CI, -2.85 to $-1.31 ; \mathrm{P}=0.47)$. Thus, the alpha-blocker+ imidafenacin group showed an equivalent effect in terms of the IPSS total score to that of the alpha-blocker only group (Fig. 4).

\section{IPSS storage symptoms score}

Three RCTs containing 690 participants (346 in the alphablocker+imidafenacin group and 344 in the alpha-blocker only group) were included. A random-effects model was used to evaluate changes between the alpha-blocker+imidafenacin group and the alpha-blocker only group, showing an $\mathrm{MD}$ of -0.99 (95\% CI, -2.07 to $0.10 ; \mathrm{P}=0.07$ ). This result shows that the 2 groups had no significant differences in the IPSS storage symptoms score (Fig. 4).

\section{IPSS voiding symptoms score}

Three RCTs including 690 participants (346 in the alphablocker+imidafenacin group and 344 in the alpha-blocker only group) were included. A random-effects model was chosen to 
Table 2. Quality assessment of individual study

\begin{tabular}{lcccccccc}
\hline Study & $\begin{array}{c}\text { Allocation } \\
\text { sequence } \\
\text { generation }\end{array}$ & $\begin{array}{c}\text { Allocation } \\
\text { concealment }\end{array}$ & Blinding & $\begin{array}{c}\text { Loss to } \\
\text { follow-up }\end{array}$ & $\begin{array}{c}\text { Calculation of } \\
\text { sample size }\end{array}$ & $\begin{array}{c}\text { Statistical } \\
\text { analysis }\end{array}$ & $\begin{array}{c}\text { ITT analysis } \\
\text { quality }\end{array}$ \\
\hline Cho et al. 2017 [17] & A & A & A & 0 & YES & ANCOVA & NO & A \\
Takeda et al. 2013 [13] & A & A & A & 0 & YES & ANCOVA & NO & A \\
Yamanishi et al. 2017 [20] & A & A & A & 6 & YES & ANCOVA & NO & A \\
Yokoyama et al. 2015 [18] & A & A & B & 2 & YES & ANCOVA & NO & B \\
\hline
\end{tabular}

A, all quality criteria met (adequate): low risk of bias; B, one or more of the quality criteria only partly met (unclear): moderate risk of bias; C, one or more criteria not met (inadequate or not used): high risk of bias; ITT, intention-to-treat analysis; ANCOVA, analysis of covariance.

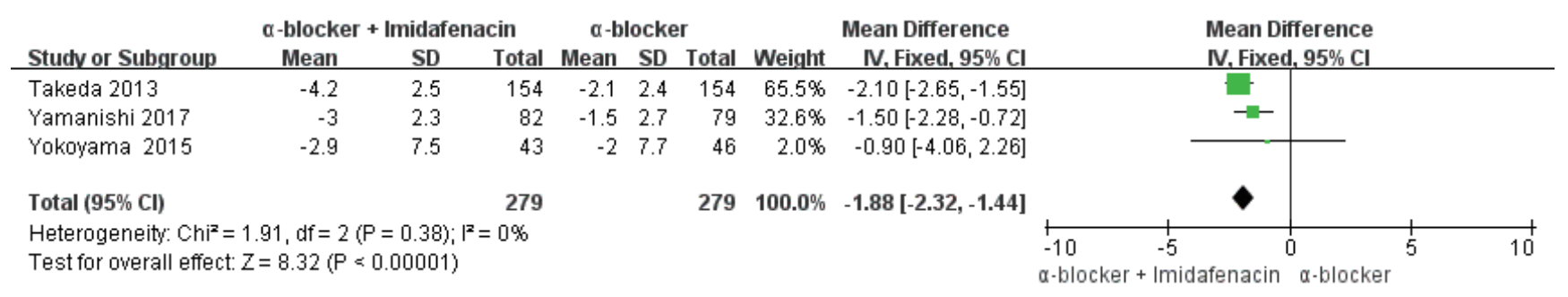

Fig. 3. Forest plots showing changes between the 2 groups in the Overactive Bladder Symptom Score. SD, standard deviation; IV, inverse variance; $\mathrm{CI}$, confidence interval; $\mathrm{df}$, degrees of freedom.

evaluate changes between the alpha-blocker and imidafenacin group and the alpha-blocker only group, showing an $\mathrm{MD}$ of -0.34 ( $95 \% \mathrm{CI},-0.94$ to $1.61, \mathrm{P}=0.60)$. Thus, the alpha-blocker+ imidafenacin group showed an equivalent effect in terms of the IPSS voiding symptom score to that of the imidafenacin group (Fig. 4).

\section{IPSSQOL}

Four RCTs containing 779 participants (389 in the alphablocker+imidafenacin group and 390 in the alpha-blocker only group) were included. A random-effects model was used to evaluate changes between the alpha-blocker+imidafenacin group and the alpha-blocker only group, showing an $\mathrm{MD}$ of -1.49 (95\% CI, -3.65 to $0.67 ; \mathrm{P}=0.18$ ). This finding demonstrates that the 2 groups were similar in terms of the IPSS QoL (Fig. 4).

\section{Safety}

PVR

Four RCTs containing 779 participants (389 in the alphablocker+imidafenacin group and 390 in the alpha-blocker only group) analyzed PVR. A random-effects model was adopted to evaluate changes between the alpha-blocker+imidafenacin group and the alpha-blocker only group, showing an MD of 6.88 ( $95 \%$ CI, 0.07 to $13.68 ; \mathrm{P}=0.05$ ). This result shows that the alpha-blocker+imidafenacin group had equivalent outcomes to the alpha-blocker only group in terms of PVR (Fig. 5).

\section{Qmax}

Three RCTs containing 690 participants (346 in the alphablocker+imidafenacin group and 344 in the alpha-blocker group) were included. A random-effects model was used to evaluate changes between the two groups, showing an MD of 0.55 (95\% CI, -1.16 to $2.26, \mathrm{P}=0.53$ ). Thus, there was no significant difference in terms of Qmax between the 2 groups (Fig. 5).

\section{DISCUSSION}

In recent years, increasingly many BPH patients have shown symptoms of $\mathrm{OAB}$, and alpha-blockers used alone cannot relieve the symptoms of $\mathrm{OAB}$ in these patients. Therefore, there is increasing interest in combining drugs to treat $\mathrm{OAB}$ symptoms in patients with $\mathrm{BPH}$.

The meta-analysis integrated evidence from 4 studies to evaluate the efficacy and safety of combination therapy that comprised an alpha-blocker (tamsulosin, alfuzosin, naftopidil, or silodosin), and imidafenacin (0.1 mg or $0.2 \mathrm{mg}$ twice a day), an anticholinergic agent, in $\mathrm{BPH}$ patients with $\mathrm{OAB}$. In this metaanalysis, compared with the alpha-blocker only group, the alpha- 


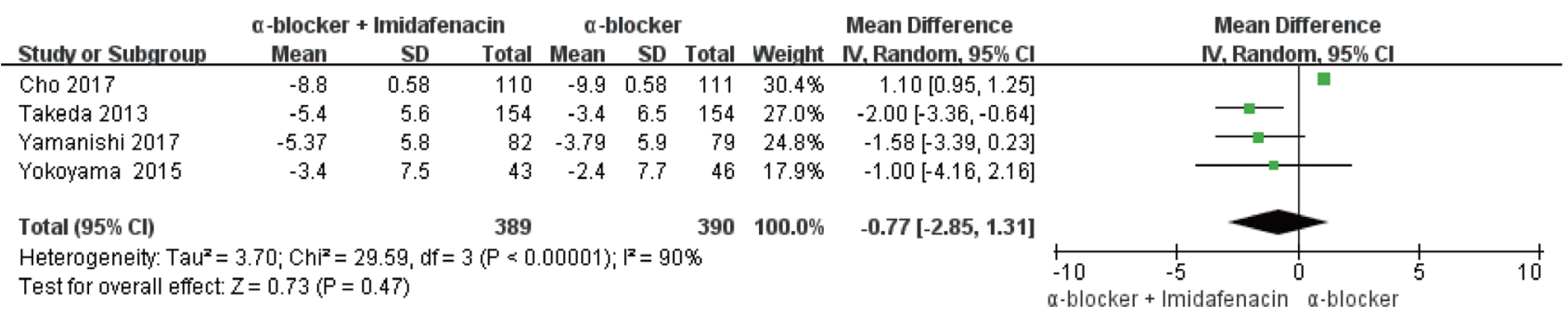

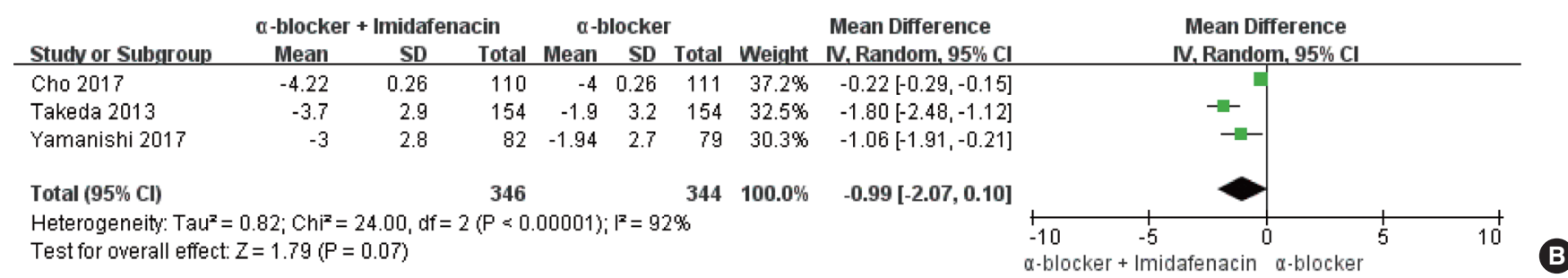

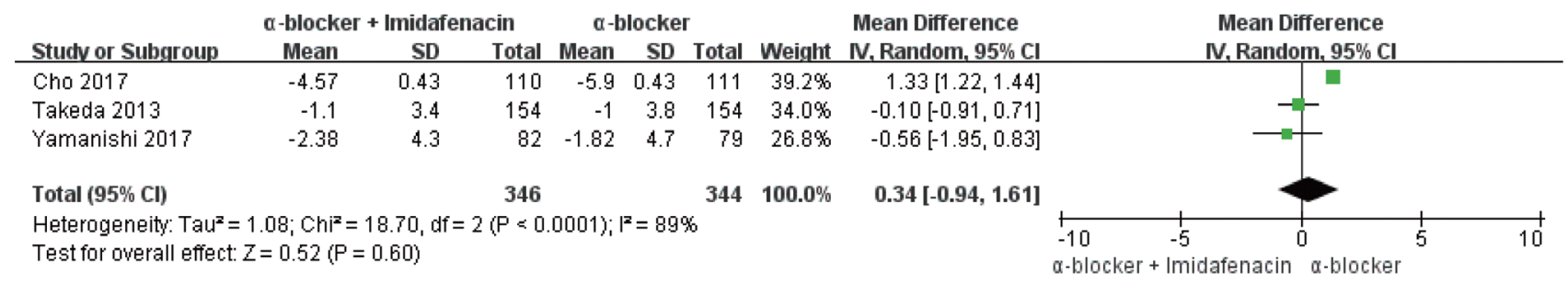

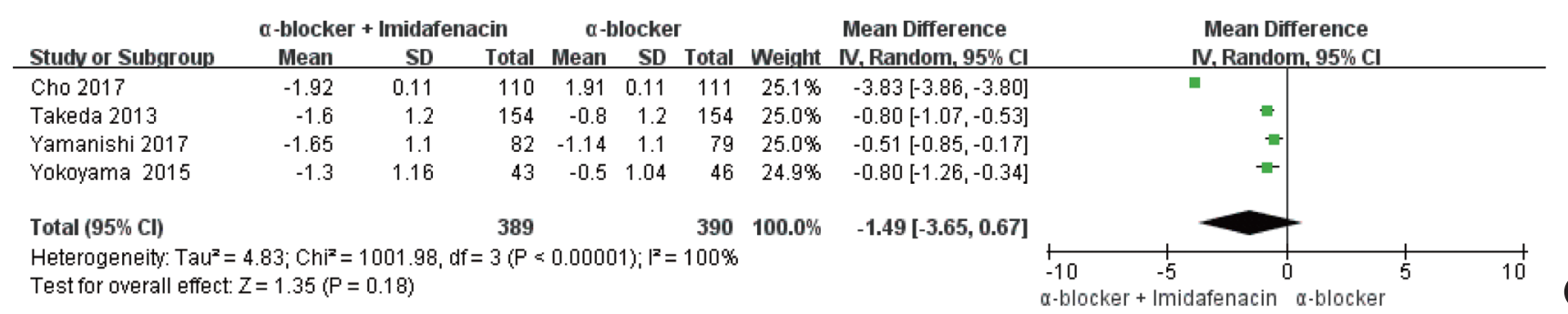

Fig. 4. Forest plots showing changes between the 2 groups in the International Prostate Symptom Score (IPSS) total score (A), the IPSS storage symptoms score (B), the IPSS voiding symptoms score (C), and the IPSS quality of life (D). SD, standard deviation; IV, inverse variance; $\mathrm{CI}$, confidence interval; $\mathrm{df}$, degrees of freedom.

blocker+imidafenacin group showed a statistically significant greater reduction in OABSS compared with the alpha-blocker only group $(\mathrm{P}<0.00001)$. The alpha-blocker+imidafenacin group showed better results in terms of improvements in $\mathrm{OAB}$ symptoms than the alpha-blocker only group up to 12 weeks in $\mathrm{BPH}$ patients. At the same time, compared with the alphablocker only group, the alpha-blocker+imidafenacin group showed equivalent results in treating $\mathrm{OAB}$ with $\mathrm{BPH}$ in terms of the IPSS total score $(\mathrm{P}=0.47)$, the IPSS storage symptoms score $(\mathrm{P}=0.07)$, the IPSS voiding symptoms score $(\mathrm{P}=0.60)$, and the IPSS QoL $(\mathrm{P}=0.18)$. In the present study, the alpha-blocker+ imidafenacin group demonstrated significant improvements from baseline in the OABSS, and the 2 group also had equivalent effects in the IPSS and other indexes related to BPH. As a result, combination therapy with an imidafenacin add-on to alpha-blocker can significantly improve the symptoms of $\mathrm{OAB}$ compared with alpha-blocker monotherapy without deteriorating urination symptoms.

The efficacy of imidafenacin was similar to those of conventional anticholinergic agents, with a favorable tolerance profile. In a rat experiment, it was potently inhibited the rhythmic contractions of the bladder and significantly increased bladder capacity [21]. It has high affinities for the M3 and M1 muscarinic receptor subtypes and a low affinity for M2 receptors [21,22]. 


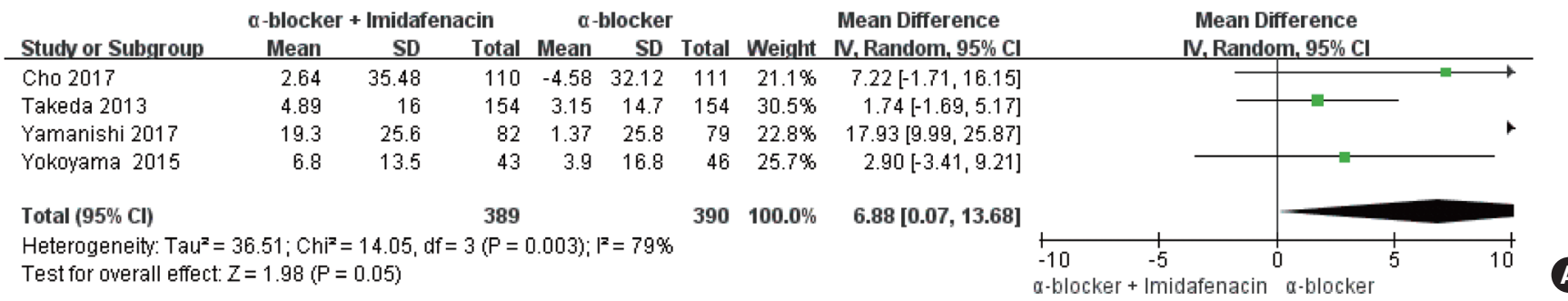

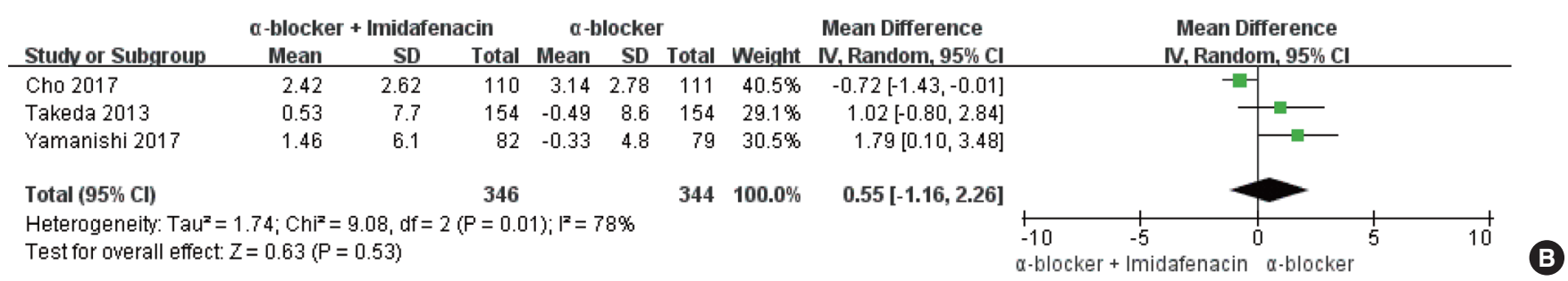

Fig. 5. Forest plots showing changes between the 2 groups in postvoid residual volume (A), maximum flow rate (B). SD, standard deviation; IV, inverse variance; $\mathrm{CI}$, confidence interval; $\mathrm{df}$, degrees of freedom.

Meanwhile, in clinical experiments, imidafenacin blocked postjunctional M3 receptors and prejunctional M1 receptors to inhibit the contractions of the detrusor to smooth muscles in humans. In addition, imidafenacin displayed a high organ selectivity for the bladder and a low selectivity for salivary gland tissues [23]. Thus, compared with other antimuscarinics, imidafenacin may produce fewer side effects. As we all know, alpha-blockers reduce voiding and storage symptoms for $\mathrm{BPH}$, but sometimes it is difficult to improve storage symptoms by using alpha-blockers alone [24-27]. Several studies have shown that the application of an anticholinergic agent with an alphablocker was effective for patients with BPH who had storage symptoms [28-32]. Meanwhile, according to the literature, adding an anticholinergic is recommended in cases of recalcitrant $\mathrm{OAB}$ [33].

We have noted that many clinicians are hesitant to use anticholinergics in clinical practice because they may exacerbate the symptoms of $\mathrm{OAB}$, particularly in patients with possible $\mathrm{BPH}$. In this meta-analysis, the alpha-blocker+imidafenacin group and alpha-blocker only group showed similar results in terms of the $\mathrm{Qmax}(\mathrm{P}=0.53)$. It is remarkable that compared with the alpha-blocker group, the alpha-blocker+imidafenacin group had a similar effect regarding PVR in treating OAB with $\mathrm{BPH}(\mathrm{P}=0.05)$. These results demonstrate that for men with $\mathrm{OAB}$, imidafenacin add-on treatment with an alpha-blocker was safe. Although the 4 RCTs reported adverse events, no notable events were reported including urinary retention or di- minished cognitive function. The results of safety and tolerability in this meta-analysis demonstrated that alpha-blocker+ imidafenacin combination treatment was well-tolerated. It is worth mentioning that the combination of alpha-blocker and imidafenacin did not lead to significant improvements in IPSS storage symptoms. Two possible reasons for this are: (1) the RCTs that we reviewed had short intervention periods, and it may take a longer time to achieve significant improvement, and (2) we primarily evaluated the additional benefits of the addition of imidafenacin; if a different dose of imidafenacin had been used, different results would be expected.

Compared with salivary gland tissue, imidafenacin, as a short circulating half-life anticholinergic, has higher selectivity for bladder tissue. Wada et al. [34] showed that imidafenacin reduced the frequency of nocturia and improved quality of sleep. Two reasons may explain how imidafenacin decreases nocturnal urine volume: (1) it could inhibit the bladder afferent nerves directly to affect nocturnal urine volume, or (2) it may improve sleep quality indirectly. Research has shown that the use of sleeping pills by patients with BPH could decrease the frequency of nocturia. In this study, the results regarding PVR were analogous between the 2 groups [35], which may be related to the dose of the drug. In the alpha-blocker+imidafenacin group, the risk of urinary retention may be reduced with a lower dose than usual. Simultaneously, compared with other anticholinergic drugs, imidafenacin has a shorter half-life ( 2.9 hours) and lower accumulation, and it did not cause urinary retention at a 
dose of $0.2 \mathrm{mg} /$ day $[23,36]$. However, the results for Qmax showed that the combination of alpha-blocker+imidafenacin did not reduce voiding pressure and flow rates. A massive release of acetylcholine could reduce the effect of imidafenacin [37]. Therefore, high anticholinergic doses damage bladder contractility. Furthermore, compared with alpha-blocker monotherapy, add-on imidafenacin treatment showed better results in terms of improvements in OAB symptoms for BPH patients in a long-term evaluation [38]. Of course, much more research is needed to demonstrate the above points conclusively.

This meta-analysis included 4 RCTs and focused on the efficacy and safety of alpha-blocker+imidafenacin combination treatment on $\mathrm{OAB}$ associated with $\mathrm{BPH}$ in men compared with alpha-blocker monotherapy. Compared with previous studies, our study had some advantages; principally, the data were derived from randomized, double-blind, controlled trials. Furthermore, we draw conclusions with meaningful clinical significance based on the most up-to-date, accurate data. However, this study also has some limitations, which reflect common limitations of systematic reviews and meta-analyses. First of all, this article did not include numerous RCTs or unpublished studies, and the availability of limited evidence may have affected the study quality. Second, different types of alpha-blockers were used, which may have affected the results, and people in different regions have different reactions to imidafenacin, which also affects the test results to some extent. Furthermore, urodynamic examinations are an important assessment method, the RCTs included in the current study did not analyze a full range of urodynamic parameters. Furthermore, these 4 RCTs mainly investigated populations in Asia. The result of PVR is precisely on the threshold of statistical significance, and we need more appropriate high-quality trials to improve the accuracy of the results.

In conclusion, in $\mathrm{BPH}$ patients with persistent $\mathrm{OAB}$ symptoms, combination therapy with imidafenacin add-on to an alpha-blocker showed improved efficacy and safety compared with an alpha-blocker alone.

\section{AUTHOR CONTRIBUTION STATEMENT}

Conceptualization: $Y C$

Data curation: $N W, L L$

Formal analysis: $T C$

Funding acquisition: $Y Z$

Methodology: TC
Project administration: $Y C$

Visualization: $L L, Z Z$

Writing-original draft: $T C, N W$

Writing-review \& editing: ZZ, YZ, YC

\section{REFERENCES}

1. Bosch JL, Hop WC, Kirkels WJ, Schröder FH. Natural history of benign prostatic hyperplasia: appropriate case definition and estimation of its prevalence in the community. Urology 1995;46(3 Suppl A):34-40.

2. Boyle P, Robertson C, Mazzetta C, Keech M, Hobbs FD, Fourcade R, et al. The prevalence of lower urinary tract symptoms in men and women in four centres. The UrEpik study. BJU Int 2003;92:409-14.

3. Witjes WP, de la Rosette JJ, Donovan JL, Peters TJ, Abrams P, Kay HE, et al. The International Continence Society "Benign Prostatic Hyperplasia” Study: international differences in lower urinary tract symptoms and related bother. J Urol 1997;157:1295-300.

4. Athanasopoulos A, Gyftopoulos K, Giannitsas K, Fisfis J, Perimenis P, Barbalias G. Combination treatment with an alpha-blocker plus an anticholinergic for bladder outlet obstruction: a prospective, randomized, controlled study. J Urol 2003;169:2253-6.

5. Lee KS, Choo MS, Kim DY, Kim JC, Kim HJ, Min KS, et al. Combination treatment with propiverine hydrochloride plus doxazosin controlled release gastrointestinal therapeutic system formulation for overactive bladder and coexisting benign prostatic obstruction: a prospective, randomized, controlled multicenter study. J Urol 2005;174(4 Pt 1):1334-8.

6. Kaplan SA, Roehrborn CG, Chancellor M, Carlsson M, Bavendam T, Guan Z. Extended-release tolterodine with or without tamsulosin in men with lower urinary tract symptoms and overactive bladder: effects on urinary symptoms assessed by the International Prostate Symptom Score. BJU Int 2008;102:1133-9.

7. Bae JH, Kim SO, Yoo ES, Moon KH, Kyung YS, Kim HJ. Efficacy and safety of low-dose propiverine in patients with lower urinary tract symptoms/benign prostatic hyperplasia with storage symptoms: a prospective, randomized, single-blinded and multicenter clinical trial. Korean J Urol 2011;52:274-8.

8. Yokoyama O, Homma Y, Yamaguchi O. Imidafenacin, an antimuscarinic agent, improves nocturia and reduces nocturnal urine volume. Urology 2013;82:515-20.

9. Smith AL, Wein AJ. Outcomes of pharmacological management of nocturia with non-antidiuretic agents: does statistically significant equal clinically significant? BJU Int 2011;107:1550-4.

10. Jacobsen SJ, Jacobson DJ, Girman CJ, Roberts RO, Rhodes T, Guess 
HA, et al. Natural history of prostatism: risk factors for acute urinary retention. J Urol 1997;158:481-7.

11. Chapple CR, Smith D. The pathophysiological changes in the bladder obstructed by benign prostatic hyperplasia. Br J Urol 1994;73: 117-23.

12. Nishizawa O, Yamaguchi O, Takeda M, Yokoyama O; TAABO Study Group. Randomized controlled trial to treat benign prostatic hyperplasia with overactive bladder using an alpha-blocker combined with anticholinergics. Low Urin Tract Symptoms 2011;3:2935.

13. Takeda M, Nishizawa O, Gotoh M, Yoshida M, Takahashi S, Masumori N. Clinical efficacy and safety of imidafenacin as add-on treatment for persistent overactive bladder symptoms despite a-blocker treatment in patients with BPH: the ADDITION study. Urology 2013;82:887-93.

14. Gong M, Dong W, Huang G, Gong Z, Deng D, Qiu S, et al. Tamsulosin combined with solifenacin versus tamsulosin monotherapy for male lower urinary tract symptoms: a meta-analysis. Curr Med Res Opin 2015;31:1781-92.

15. Vader JP. Randomised controlled trials: A user's guide. BMJ 1998; 317:1258.

16. DerSimonian R, Laird N. Meta-analysis in clinical trials. Control Clin Trials 1986;7:177-88.

17. Cho S, Kwon SS, Lee KW, Yoo TK, Shin DG, Kim SW, et al. A multicenter real-life study of the efficacy of an alpha-blocker with or without anticholinergic agent (imidafenacin) treatment in patients with lower urinary tract symptoms/benign prostatic hyperplasia and storage symptoms. Int J Clin Pract 2017;71.

18. Yokoyama O, Tsujimura A, Akino H, Segawa N, Tamada S, Oguchi $\mathrm{N}$, et al. Add-on anticholinergic therapy for residual nocturia in patients with lower urinary tract symptoms receiving al-blocker treatment: a multi-centre, prospective, randomised study. World J Urol 2015;33:659-67.

19. Xu Y, Liu R, Liu C, Cui Y, Gao Z. Meta-analysis of the efficacy and safety of mirabegron add-on therapy to solifenacin for overactive bladder. Int Neurourol J 2017;21:212-9.

20. Yamanishi T, Asakura H, Seki N, Tokunaga S. Efficacy and safety of combination therapy with tamsulosin, dutasteride and imidafenacin for the management of overactive bladder symptoms associated with benign prostatic hyperplasia: A multicenter, randomized, open-label, controlled trial (DIrecT Study). Int J Urol 2017;24:52531.

21. Kobayashi F, Yageta Y, Yamazaki T, Wakabayashi E, Inoue M, Segawa M, et al. Pharmacological effects of imidafenacin (KRP-197/ ONO-8025), a new bladder selective anti-cholinergic agent, in rats.
Comparison of effects on urinary bladder capacity and contraction, salivary secretion and performance in the Morris water maze task. Arzneimittelforschung 2007;57:147-54.

22. Murakami S, Yoshida M, Iwashita H, Otani M, Miyamae K, Masunaga K, et al. Pharmacological effects of KRP-197 on the human isolated urinary bladder. Urol Int 2003;71:290-8.

23. Yamazaki T, Muraki Y, Anraku T. In vivo bladder selectivity of imidafenacin, a novel antimuscarinic agent, assessed by using an effectiveness index for bladder capacity in rats. Naunyn Schmiedebergs Arch Pharmacol 2011;384:319-29.

24. Kawabe K. Latest frontiers in pharmacotherapy for benign prostatic hyperplasia. Yakugaku Zasshi 2006;126 Spec no:199-206.

25. Narayan P, Evans CP, Moon T. Long-term safety and efficacy of tamsulosin for the treatment of lower urinary tract symptoms associated with benign prostatic hyperplasia. J Urol 2003;170(2 Pt 1):498-502.

26. Lepor H. Phase III multicenter placebo-controlled study of tamsulosin in benign prostatic hyperplasia. Tamsulosin Investigator Group Urology 1998;51:892-900.

27. Abrams P, Schulman CC, Vaage S. Tamsulosin, a selective alpha 1cadrenoceptor antagonist: a randomized, controlled trial in patients with benign prostatic 'obstruction' (symptomatic BPH). The European Tamsulosin Study Group. Br J Urol 1995;76:325-36.

28. Yamaguchi O, Kakizaki H, Homma Y, Takeda M, Nishizawa O, Gotoh M, et al. Solifenacin as add-on therapy for overactive bladder symptoms in men treated for lower urinary tract symptoms-ASSIST, randomized controlled study. Urology 2011;78:126-33.

29. Kaplan SA, McCammon K, Fincher R, Fakhoury A, He W. Safety and tolerability of solifenacin add-on therapy to $\alpha$-blocker treated men with residual urgency and frequency. J Urol 2013;189(1 Suppl): S129-34.

30. Kaplan SA, Roehrborn CG, Rovner ES, Carlsson M, Bavendam T, Guan Z. Tolterodine and tamsulosin for treatment of men with lower urinary tract symptoms and overactive bladder: a randomized controlled trial. JAMA 2006;296:2319-28.

31. Chapple C, Herschorn S, Abrams P, Sun F, Brodsky M, Guan Z. Tolterodine treatment improves storage symptoms suggestive of overactive bladder in men treated with alpha-blockers. Eur Urol 2009;56:534-41.

32. Shi H, Chen H, Zhang Y, Cui Y. The efficacy and safety of Vibegron in treating overactive bladder: A systematic review and pooled analysis of randomized controlled trials. Neurourol Urodyn 2020; 39:1255-63.

33. Homma Y, Gotoh M, Kawauchi A, Kojima Y, Masumori N, Nagai A, et al. Clinical guidelines for male lower urinary tract symptoms 
and benign prostatic hyperplasia. Int J Urol 2017;24:716-29.

34. Wada N, Watanabe M, Kita M, Matsumoto S, Osanai H, Yamaguchi S, et al. Effect of imidafenacin on nocturia and sleep disorder in patients with overactive bladder. Urol Int 2012;89:215-21.

35. Matsuta Y, Yusup A, Tanase K, Ishida H, Akino H, Yokoyama O. Melatonin increases bladder capacity via GABAergic system and decreases urine volume in rats. J Urol 2010;184:386-91.

36. Bunniran S, Davis C, Kristy R, Ng D, Schermer CR, Uribe C, et al. A prospective study of elderly initiating mirabegron versus antimuscarinics: patient reported outcomes from the Overactive Bladder Satisfaction Scales and other instruments. Neurourol Urodyn
2018;37:177-85.

37. Andersson KE, Yoshida M. Antimuscarinics and the overactive detrusor--which is the main mechanism of action? Eur Urol 2003;43: 1-5.

38. Yamanishi T, Asakura H, Seki N, Tokunaga S. A 52-week multicenter randomized controlled study of the efficacy and safety of add-on dutasteride and imidafenacin to tamsulosin in patients with benign prostatic hyperplasia with remaining overactive bladder symptoms (DIrecT study). Low Urin Tract Symptoms 2019;11: 115-21. 